\title{
Harnessing autocatalytic reactions in polymerization and depolymerization
}

\author{
Rajeev KumarD, Zening Liu, Brad Lokitz, Jihua Chen, Jan-Michael Carrillo, Jacek Jakowski, C. Patrick Collier(D, \\ Scott Retterer, Rigoberto Advincula, Center for Nanophase Materials Sciences, Oak Ridge National Laboratory, Oak Ridge, TN 37831, USA \\ Address all correspondence to Rajeev Kumar at kumarr@ornl.gov
}

Rigoberto Advincula was an editor of this journal during the review and decision stage. For the MRS Communications policy on review and publication of manuscripts authored by editors, please refer to http://www.mrs.org/editor-manuscripts/

(Received 9 March 2021; accepted 21 June 2021; published online: 12 July 2021)

\begin{abstract}
Autocatalysis and its relevance to various polymeric systems are discussed by taking inspiration from biology. A number of research directions related to synthesis, characterization, and multi-scale modeling are discussed in order to harness autocatalytic reactions in a useful manner for different applications ranging from chemical upcycling of polymers (depolymerization and reconstruction after depolymerization), self-generating micelles and vesicles, and polymer membranes. Overall, a concerted effort involving in situ experiments, multi-scale modeling, and machine learning algorithms is proposed to understand the mechanisms of physical and chemical autocatalysis. It is argued that a control of the autocatalytic behavior in polymeric systems can revolutionize areas such as kinetic control of the self-assembly of polymeric materials, synthesis of self-healing and self-immolative polymers, as next generation of materials for a sustainable circular economy.
\end{abstract}

\section{Introduction}

Autocatalytic reactions play central roles in various physical phenomena and industrial processes such as self-replication of biological polymers ${ }^{[1,2]}$ and polymerization with moving reaction zones (known as frontal polymerization), ${ }^{[3,4]}$ respectively. By definition, these reactions involve autoacceleration of their rates due to products (autocatalysts) of the reactions. Autocatalysis ${ }^{[5]}$ requires acceleration of forward reaction rates due to the products (Fig. 1). Kinetics of autocatalytic reactions can be quantified in terms of the time required for the onset of autoacceleration (induction period), time and nonlinear rate of the reaction after the onset of autoacceleration and before reaching a saturation period. Acceleration of the forward reaction rates can be realized by using physical processes favoring the formation of products and autocatalysis may not require any explicit chemical catalyst in these reactions. Roughly, autocatalysis can be categorized into physical and chemical autocatalysis based on whether autoacceleration

This manuscript has been authored by UT-Battelle, LLC, under contract DE-AC05-00OR22725 with the US Department of Energy (DOE). The US government retains and the publisher, by accepting the article for publication, acknowledges that the US government retains a non-exclusive, paid-up, irrevocable, worldwide license to publish or reproduce the published form of this manuscript, or allow others to do so, for US government purposes. DOE will provide public access to these results of federally sponsored research in accordance with the DOE Public Access Plan (http://energy.gov/downloads/doe-public-access-plan. is a result of either physical or chemical (chemistry related) processes, respectively. An example of physical autocatalysis involves polymerization-induced self-assembly ${ }^{[6]}$ (PISA) of two monomers in a common solvent so that as the polymerization proceeds, solubility of copolymers in the solvent drives self-assembly and the rate of polymerization can be accelerated in certain cases due to local increase in concentration of reacting monomers. Key challenges in controlling and designing autocatalytic reactions lie in developing a deep understanding of the mechanism of autoacceleration, designing autocatalytic sets and cycles, harnessing chemical instabilities ${ }^{[7]}$ and pattern formation. New characterization methods and approaches capable of understanding chemical kinetics, its autoacceleration, and other physical processes (such as pattern formation due to chemical transformations) can provide key advances for designing autocatalytic reactions relevant to polymerization, depolymerization, and related self-assembly of polymers.

Autocatalysis has deep connections to machine learning algorithms ${ }^{[8]}$ built upon the notion of graphs and networks. Most of the algorithms are designed to learn non-linear relations among outputs and inputs using activation functions and weights. In fact, it can be shown ${ }^{[9]}$ that various mathematical functions representing the activation and the weights can be written down by using autocatalysis as an underlying phenomena, where a non-linear relation between the rate of forward reaction and input variables exists. In order to develop better algorithms and understand instabilities in systems far-from-equilibrium, ${ }^{[7]}$ it is imperative to develop a better 

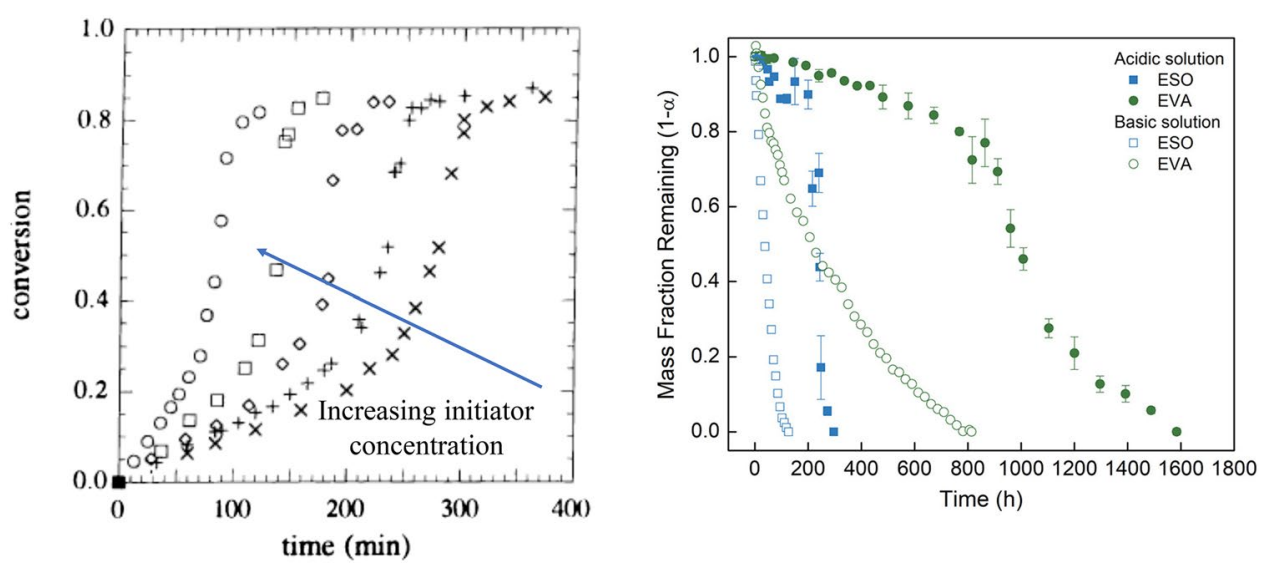

Figure 1. Typical kinetic behavior observed in autocatalysis, where a deviation from first-order rate of reaction is observed. Two examples are shown here. Left panel shows the kinetics of methylmethacrylate polymerization with different initiator concentrations. Right panel shows the kinetics of hydrolytic degradation of bio-based epoxy resins, prepared through anhydride curing of epoxidized vanillic acid (EVA, a product of lignin depolymerization) and epoxidized soybean oil (ESO). In general, autocatalysis shows an induction period, followed by autoaccelerated (non-linear) forward rates of reactions and a saturation period. Left and right panels are adapted from Refs. 16 and 18 , respectively, with permissions.

understanding of autocatalysis. With recent advances ${ }^{[10,11]}$ in polymer informatics and developments in natural language processing tools, chemical kinetics databases dedicated to polymerization and depolymerization are expected to usher in near future. These databases and neural networks, trained on models capable of describing autocatalysis (e.g., by Lotka-Volterra equations ${ }^{[12,13]}$ ), can be used to understand various aspects of autocatalysis in polymerizations. ${ }^{[14-16]}$ Furthermore, many decomposition ${ }^{[17,18]}$ reactions such as those involved in photooxidation are autocatalytic in nature and in certain cases, ${ }^{[19]}$ external forces such as fluid flow can be used to further accelerate various reactions. A detailed understanding of autoacceleration in these reactions will be useful for designing the next generation of energy-efficient chemical upcycling protocols, ${ }^{[18]}$ photo-initiated polymerizations ${ }^{[20]}$ relevant to $3 \mathrm{D}$ printing processes, and design of supramolecular polymeric materials such as self-generating micelles. ${ }^{[6]}$

In this prospective article, we discuss the most interesting directions of research relevant to polymers based on the concepts of physical and chemical autocatalysis. Development of multi-scale models capable of treating chemical reactions and polymeric aspects is proposed in order to understand mechanism of autocatalysis by simulating different reaction pathways. A rational design of the most efficient reaction pathway requires a merger of the proposed experimental efforts concerning the rate of forward reactions and multi-scale models using neural networks. In the following, we present three areas of research focused on (1) autocatalysis in polymer synthesis and its relevance to depolymerization, (2) biphasic autocatalysis relevant to reactions near oil-water interfaces, and (3) understanding autocatalysis using multi-scale models, which can simulate chemical and physical processes at different lengths and time scales.

\section{Status quo and prospective directions Autocatalysis in kinetics of polymerization: breakdown of equal reactivity hypothesis}

Autoacceleration, a common signature of autocatalysis, in freeradical polymerization is well documented in the literature and is referred to as either "Norrish-Smith" ${ }^{[14]}$ or "Trommsdorff" [15] (gel) effect. The effect has been observed in the polymerization of methyl methacrylate (MMA) (see Fig. 1), styrene, vinyl acetate, methyl acrylate, other acrylates and methacrylate-based monomers. Experimentally, autoacceleration in the kinetics of free-radical polymerization can be identified by plotting monomer conversion as a function of time. Without any kind of autoacceleration, first-order reaction kinetics is expected for a free-radical polymerization. However, when autoacceleration occurs, deviation from first-order kinetics occurs and a significant increase in the slope of monomer conversion versus time is observed.

An explanation for the autoacceleration was put forth by Tulig and Tirrell ${ }^{[21]}$ based on theories for polymer diffusion in solutions. The explanation was based on an observation that autoacceleration results from an increase in solution viscosity rather than heat production ${ }^{[14]}$ when monomer reaches a critical conversion value in bulk or concentrated free-radical polymerizations. As solution viscosity depends on molecular weight of the polymers being polymerized, the critical conversion of monomers can be approximated by the conversion, which leads to an onset of "entangled" polymers in polymerization. Such an explanation of the autoacceleration in free-radical polymerization is based on the underlying assumption that these reactions are diffusion controlled. Using the Smoluchowski equation for rate of diffusion-controlled reactions and the reptation model for entangled polymers, Tulig and Tirrell ${ }^{[21]}$ showed that the 
termination rate constant $\left(k_{t}\right)$ in free-radical polymerization should be dependent on the degree of polymerization $(N)$ and concentration of polymers $(c)$ in solution by the relation $k_{t} \sim 1 / N^{2} c^{1.75}$. It should be noted that such a dependence of the termination rate implies that all of free radicals are not equally reactive and is in contrast to Flory's equal reactivity hypothesis. ${ }^{[22]}$ We should point out that the autoacceleration is a hallmark of free-radical polymerizations in concentrated solutions, where equal reactivity hypothesis is shown to be inapplicable. A number of other models ${ }^{[23]}$ based on different dependencies of the diffusion constant on chain length and polymer concentration e.g., using free volume models, has been developed with additional parameters.

As free-radical polymerization leads to chains of different degrees of polymerizations, effects of chain distribution or dispersity on autoacceleration were considered by O'Shaughnessy et al. ${ }^{[24]}$ These investigations showed that diffusion of long entangled polymers is slowed down so much that short-long chain recombination is the fastest termination step during the autoacceleration. As a direct consequence of this, polymer production rate during autoacceleration was predicted ${ }^{[24]}$ to be independent of the free-radical production rate in contrast to square root dependence on the radical production rate in Flory-Schulz's theory of free-radical polymerization based on the equal reactivity principle. A weak dependence of the polymer production rate on chain transfer was conjectured during autoacceleration. However, Torkelson and coworkers looked ${ }^{[16]}$ at bulk MMA and styrene polymerizations and added chain transfer agents and/or used higher initiator concentrations to delay or eliminate chain entanglements and the polymerizations still exhibited autoacceleration behavior. These works highlighted that the onset of the autoacceleration may be unrelated to the formation of entangled polymers and pointed out a need to understand the roles of translational self-diffusion of chains, segmental diffusion, and mutual diffusion of chains as well as reacting monomers in affecting kinetics of autoacceleration. More recently, Wöll and coworkers ${ }^{[25]}$ used fluorescent probes and fluorescently labeled poly(MMA) chains of different molecular weights to probe the role of chain diffusion on autoacceleration during bulk MMA polymerization reactions. The experimentally determined diffusion rate coefficients of the probes led the authors to conclude that the polymerization reaction was heterogeneous and it had a significant impact on the termination rate constants. ${ }^{[25]}$ If not properly accounted for, the Trommsdorff effect can result in undesirable final material properties and/or reactor failure due to the exothermic nature of autoacceleration. Recently, Zhan and coworkers ${ }^{[26,27]}$ have looked to take advantage of the Trommsdorff effect. They investigated the effect of shear rate on the Trommsdorff effect during the bulk polymerization of MMA and were able to use the results to produce PMMA with improved properties (compared to commercial PMMA) by using reactive extrusion techniques. ${ }^{[26]}$ The authors expanded on their previous work ${ }^{[26]}$ to prepare methylmethacrylate-styrene copolymers in an inverted two-stage extruder by continuously feeding the co monomers into the extruder at the onset of the Trommsdorff effect to produce a copolymer with the desired physical properties. ${ }^{[27]}$

Moving forward, experiments such as small-angle neutron scattering (SANS) and neutron spin echo coupled with new molecular theories could provide for a more complete picture of autoacceleration so that it might be used to improve industrial polymerization processes and material properties. For example ${ }^{[28,29]}$ SANS (in combination with ${ }^{1} \mathrm{H}-\mathrm{NMR}$ ) has been used to study the kinetics of polymerization and detect aggregation in solution. As the scattering from the polymers depends on their concentration in solution, the scattering intensity $(I)$ at a particular wave vector $(q)$ and time ( $t$ ) during polymerization can be fitted by $I(q, t) \sim \phi(t) n_{\text {agg }}(t) V_{w}(t) P_{\text {agg }}(q, t)$, where $\phi(t)$ is the time-dependent polymer concentration, $n_{\text {agg }}(t)$ is number of aggregates, $V_{w}(t)$ is molecular volume of a growing chain, and $P_{\text {agg }}(q, t)$ is the form factor of the aggregate. For small-scale aggregates, expected near the initiation step of the polymerization, $P_{\text {agg }}(q, t)$ can be approximated by the Guinier's formula and has to be approximated by other functions for intermediate and large-scale aggregates. Here, molecular models can help in predicting the scattering intensity resulting from polymerization-induced aggregation in solution. In the absence of detailed molecular models, ad hoc expressions of the form factor have been used to gain insights into the aggregation state during the initiation and propagation stages of polymerization. In this regard, work by Niu et al. ${ }^{[28,29]}$ is noteworthy focusing on the polymerization of butadiene and 1-octene. Studies using SANS and neutron spin echo can provide insights into the role of aggregation and resulting heterogeneous dynamics of polymer chains for different percentages of conversion. However, macromolecular models similar to the tube model of entangled polymers (used in the work by Tulig and Tirrell ${ }^{[21]}$ ) are needed in order to understand the autoacceleration during the polymerization, viscoelastic changes, and heterogeneous dynamics in solution.

\section{Prospective direction: autocatalysis}

in the kinetics of free-radical depolymerization

Similar to polymerization, free radicals play central roles in degradation and depolymerization of polymers ${ }^{[17,18]}$. In a series of papers by Simha and coworkers ${ }^{[30-32]}$, free-radical depolymerization was described by assuming that it follows similar four steps as in polymerization: initiation/generation of radicals, propagation along a chain, chain transfer (inter- and intrachain), and termination. Based on this, random chain scission and unzipping were considered to be two main mechanisms for free-radical depolymerization. Furthermore, effects of side groups in affecting chain transfer and termination were conjectured to be significant by direct comparisons of kinetic equation-based models with experimental data for polyolefins, polyacrylates, and polystyrene. Following the lessons ${ }^{[25]}$ learned about autoacceleration in the kinetics of polymerization, we can conjecture that heterogeneity in the diffusion of active radicals and the presence of high viscosity solvents such as ionic liquids can cause autoacceleration in the kinetics of depolymerization. 
Photo-oxidation of isotactic polypropylene ${ }^{[33]}$ films partially confirms this conjecture about the autoacceleration due to heterogeneity. However, detailed studies are needed to understand molecular mechanism of autocatalysis in photo-oxidation.

\section{Prospective direction: understanding autoacceleration in reactions involving electrostatics}

A majority of the works discussed above are based on diffusioncontrolled reactions, where diffusion of reacting species controls the reaction rates. However, polymerization and depolymerization reactions may not be diffusion limited in the presence of explicit ionic charges present either on the solvents such as in ionic liquids ${ }^{[34,35]}$ or on the monomers being polymerized (e.g., in polymerized ionic liquids ${ }^{[36]}$ and polyzwitterions ${ }^{[37]}$ ). In these cases, effects of electrostatics must be taken into consideration in affecting reaction rates as predicted by Marcus's theory of electron transfer, ${ }^{[38]}$ where solvent plays a decisive role in affecting reorganization energy and reaction rates during the transfer. In addition, charge generation and recombination due to ion-pair formation and dissociation need to be considered in controlling reaction rates, especially in the presence of applied electric fields. ${ }^{[39]}$ Preliminary works ${ }^{[36]}$ related to polymerizations of charged monomers show significant effects of counterions and solvent on the reaction rates. Similarly, depolymerization of lignin ${ }^{[40]}$ and polyamide ${ }^{[41]}$ has been demonstrated to occur efficiently in the presence of ionic liquids. In the future, developing a fundamental understanding of the roles played by electrostatics on reactions relevant to polymerization and depolymerization is expected to be the foci of a number of studies. For polymerization and depolymerization involving charged monomers, viscoelastic properties of solutions containing charged polymers are expected to play significant roles in affecting the onset of autoacceleration and total time for completion of these reactions. As viscoelastic properties $^{[42]}$ of charged polymers depend on salt concentration, temperature, and $\mathrm{pH}$ of solution, these additional variables can be used to study autoacceleration in reactions involving charged polymers. We expect precisely these studies focusing on the relations between salt concentration, temperature, $\mathrm{pH}$ (in addition to solvent and initiator concentration), and the time required for the onset of autoacceleration in reactions involving charged polymers to be a major research effort in future.

\section{Prospective direction: development of in situ characterization tools for understanding depolymerization}

All of the reactions discussed so far involve solvents. However, there are a number of reactions, which involve polymers in the absence of any solvent. For example, degradation of semicrystalline polymers in the presence of ultra-violet (UV) or ionizing radiations ${ }^{[43,44]}$ has been studied extensively and also involves free radicals. Simultaneous formation of free radicals at multiple locations along polymer chains can lead to either depolymerization or crosslinking. In semi-crystalline polymers such as polyethylene, energy required for depolymerization is smaller than the energy required for electronic structure changes as measured by electron energy loss spectra, but higher than that required for reduction in crystallinity. For example, in the case of polyethylene, the crystal unit cell has been shown to deform, swell irregularly, and explode under increased electron beam interactions, as evidenced by low-dose selected area electron diffraction studies. ${ }^{[45]}$ From these observations, one would expect that the depolymerization process can happen from multiple locations simultaneously. In contrast to the semicrystalline polymers, the depolymerization of amorphous polymers is relatively less studied by electron microscopes, likely due to the poor contrast and limited features in electron diffraction patterns obtained from amorphous polymers. Especially, in situ microscopic studies on kinetics of depolymerization, in general, are not well reported.

A current-generation high-speed camera offers capability to capture nanoscale features with hundreds to thousands frames per second. ${ }^{[46,47]}$ Imaging of nanoscale features in cathode-electrolyte interface ${ }^{[46]}$ as well as in protein complexes ${ }^{[47]}$ has especially benefitted from such advances. For example, at a magnification of 105,000 and $0.83 \AA$ physical pixel size, a resolution of $1.65 \AA$ can be achieved after $9 \mathrm{~h}$; and in some optimized scenarios, $2.5 \AA$ resolution is obtained in $5 \mathrm{~min} .{ }^{[47]}$ If the number of camera pixels ( 24 megapixels) is used to multiply the physical pixel resolution then this will convert to a sampling area of 0.45 square micron. Also, a fast, pulsed laser system using a pump-probe method is required to examine femtosecond dynamics of various materials and reactions in transmission electron microscopy (TEM). ${ }^{[48]}$ These include but are not limited to the monitoring of atomic movement in soft materials such as during phase transitions or changes of biological structures in aqueous environment. Instead of using laser pulses, one may use an electrical phase modulator to drastically enhance the temporal capturing capability of a traditional TEM to nano-second or even pico-second. ${ }^{[49]}$ Recent advances in in situ TEM of gas cell and liquid cell catalytic reactions ${ }^{[50]}$ may be transferred to study depolymerization kinetics if the electron-beam damage and intrinsic poor contrast of polymers can be properly addressed.

\section{Prospective direction: depolymerization in the presence of fluid flow}

In general, external forces can be used to tailor rates of reactions. For example, rates of reactions can be changed by affecting activation barriers for the reactions using external forces such as applied electric fields, thermal gradients, and fluid flow. We envision use of fluid flow in accelerating kinetics of depolymerization as a practical and promising direction of research for future. A number of advances have been made in having a desirable control over kinetics of polymerization by using a continuous flow during polymerization. Examples include control over molecular weight (MW) and its distribution in 


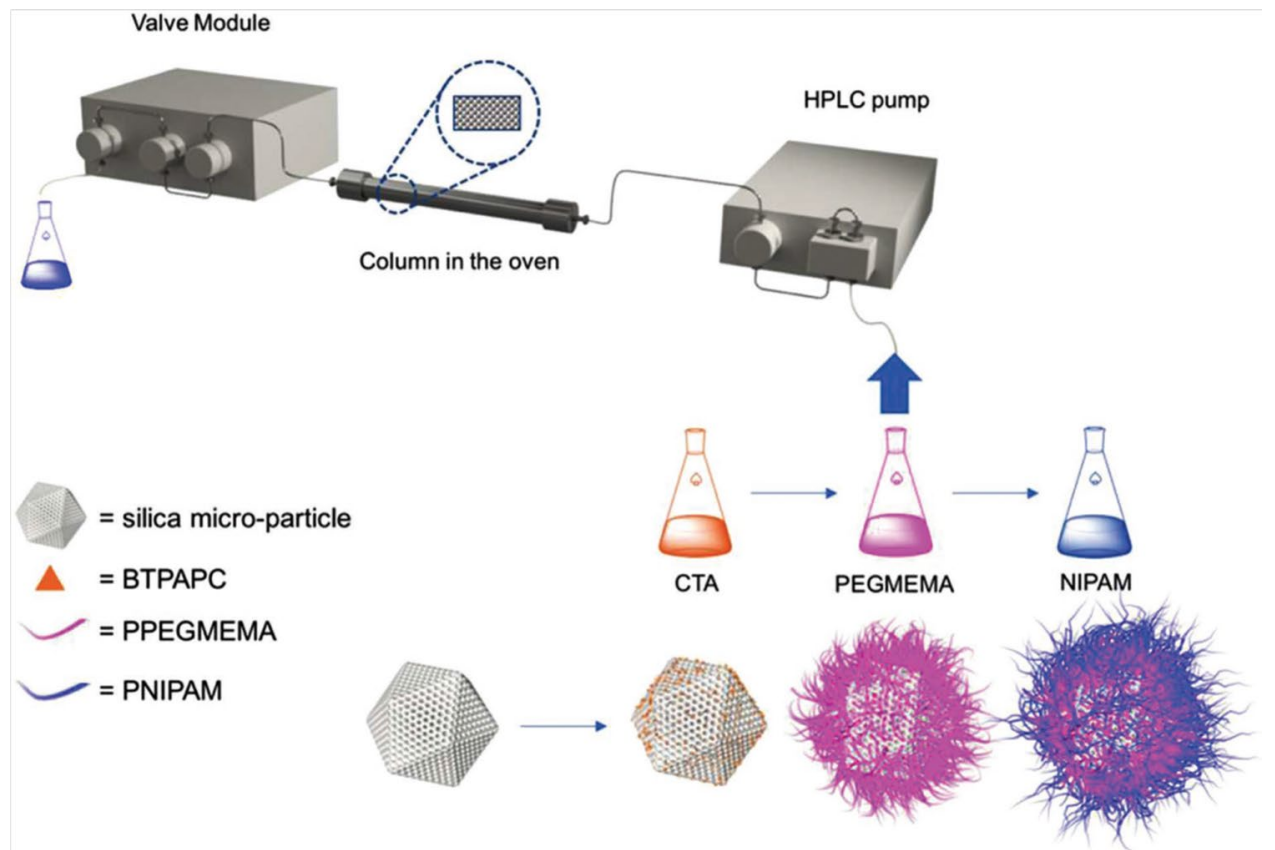

Figure 2. Continuous flow chemistry polymerization by grafting polymers (block) on a solid support media by controlling the flow rate, temperature, and pressure on grafted initiators using the RAFT mechanism. Here, CTA, NIPAM, BTPAPC, PEGMEMA, PPEGMEMA, and PNIPAM are acronyms for chain transfer agent, $N$-isopropylacrylamide, butyl (1-oxo-1-((3-(trimethoxysilyl)propyl)amino)prop-an-2-yl) carbonotrithioate, poly(ethylene glycol) methyl ether methacrylate, poly(poly(ethylene glycol) methyl ether methacrylate), and poly( $N$-isopropylacrylamide), respectively. Adapted from Ref. 52 with permissions.

free-radical polymerization, living free-radical polymerization by reversible addition fragmentation chain transfer (RAFT) in ethanol and water ${ }^{[51]}$ as well as diblock copolymerization on grafted polymers ${ }^{[52]}$ (cf. Fig. 2), ring opening polymerization (ROP) to form polyesters, living anionic polymerization of vinyl polymers, and the metathesis ring opening polymerizations. Although a better control over MW and its distribution in a flow reactor (continuous and computer-controlled) has been demonstrated for polymerization, limited progress has been made in relation to understanding kinetics of depolymerization and amplification of reaction rates. For example, controlled lignin depolymerization under continuous flow conditions has been demonstrated. ${ }^{[53]}$ By controlling the flow reactor parameters (flow rate, pressure, temperature, and column design), it is possible to obtain optimized depolymerization and autonomic degradation reactions in the next generation of self-immolative polymers. ${ }^{[54]}$ Optimizing fluid properties such as viscosity and overcoming thermal conductivity-driven limitations should be a primary focus while studying kinetics of depolymerization and the amplification of reaction rates. Using artificial intelligence (AI) and machine learning (ML) tools, it is possible to simulate, predict, and bias various systems to give specific preferred products including monomers, fragments, branching, such that these products can be separated or collected online. ${ }^{[55]}$ For example, this can be done by using neural networks for molecular and polymerization design in order to obtain targeted polymeric properties after training them to recognize quantitative structure-property relationships. As a predictive tool, it is possible to identify promising hypothetical polymer candidates, where selection can be made for monomer synthesis, polymerization, and reaction conditions under flow behavior. It is possible to use neural networks to start from a substantially limited amount of data for polymeric properties and expand or narrow the scope of explored constructs by real-time feedback from actual laboratory synthesis and thermophysical property measurements. While this has been applied in forward polymerization reactions, there is a significant interest in applying similar neural networks for designing faster depolymerization reactions on the basis of autocatalysis. For example, using programmed protocols, the molecular weight distribution (MWD) profile can be biased towards monotonic, bimodal, square, triangle, and complex MWD profiles in depolymerization reactions. In such configurations, feedback obtained about specific reaction mechanisms in depolymerization of vinyl polymers, ${ }^{[56]}$ hydrolysis of polyesters, ${ }^{[57]}$ metathesis reactions, ${ }^{[58]}$ etc. will be unique, especially when optimized in the context of a continuous flow chemistry set-up. Such feedback about reaction mechanisms can be incredibly helpful for designing recycling and upcycling protocols for polymers. ${ }^{[59]}$ Furthermore, principles learned during such studies can be a basis for starting pilot reactor and plant engineering design after scale-up. In the future, as the interest for controlled degradation of polymers becomes commercially of high interest, flow reactors will play a very important role in advancing manufacturing. 

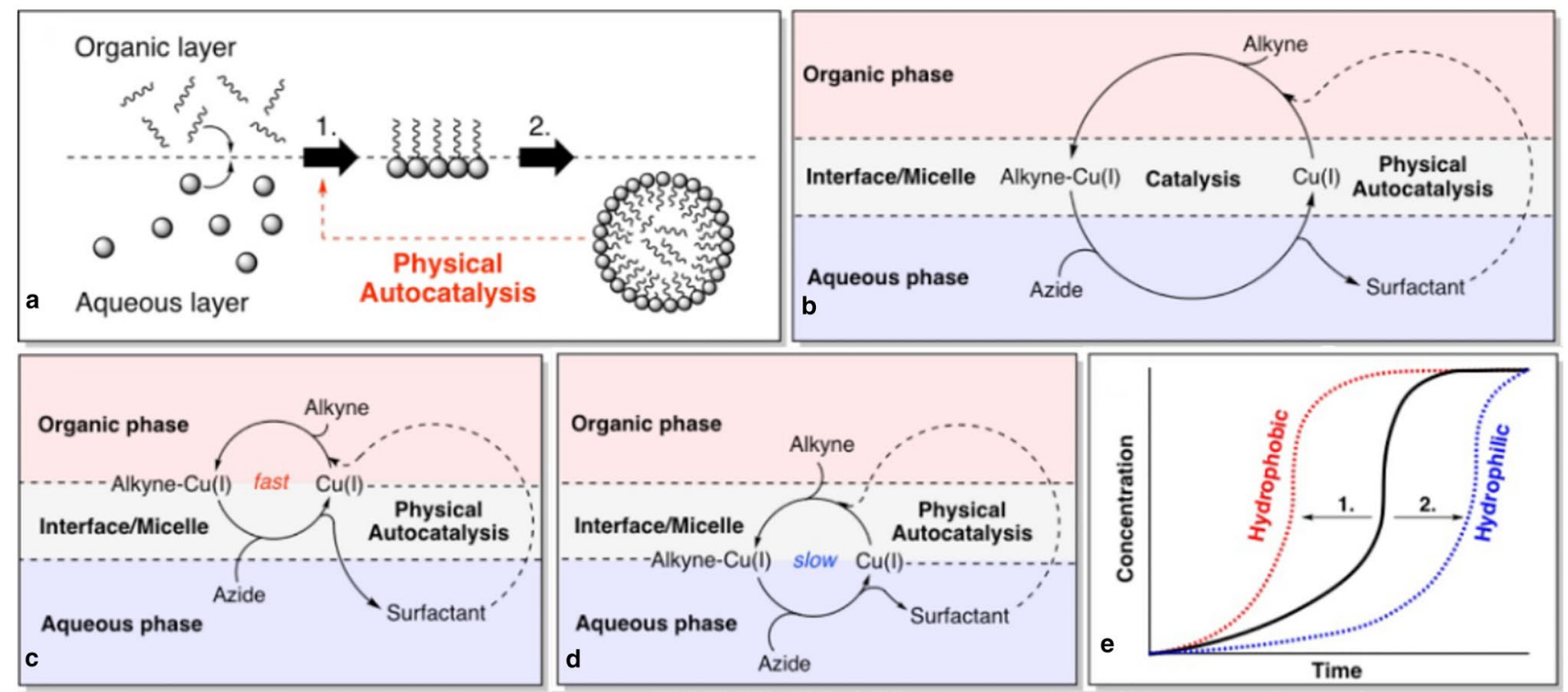

Figure 3. (a) Schematic of a typical biphasic physical autocatalysis. (b) A copper-catalyzed physical autocatalytic system, where an organic alkyne reacts first with a Cu-catalyst, then with an aqueous azide to form a surfactant. Formed micelles accelerate the reaction. (c) Using a hydrophobic Cu-ligand promotes retention of the Cu-catalyst in the organic phase and faster reaction was observed. (d) Using a hydrophilic $\mathrm{Cu}$-ligand in the system favors keeping the $\mathrm{Cu}$-catalyst in the aqueous phase and slower reaction rates were observed. (e) Summary of effect of tuning the catalyst hydrophilicity to alter reaction kinetics. (1) A hydrophobic ligand leads to shorter lag periods and higher rates while (2) a hydrophilic ligand retards the reaction. Adapted from Ref. 61 with permissions.

\section{Biphasic autocatalysis and relevance to micelles, vesicles, polymer membranes}

Autocatalysis has been proposed as the key step in the natural selection, replication, and reproduction. It has even been posed as being responsible for the origin of life. ${ }^{[1,2]}$ Similar ideas have been exercised in material sciences to develop self-generating (autopoietic) systems. For example, in the early 1990s, Luisi and collaborators first showed evidence of self-replicating micelles and vesicles starting from simple molecules. ${ }^{[60]}$ This led to an extensive research in establishing autopoietic supramolecular aggregates (including micelles, reverse micelles, vesicles, and other structures). In these studies, the underlying mechanism of self-replication is biphasic physical autocatalysis, which is the focus of this section. Biphasic physical autocatalysis refers to a reactive system having three characteristics (cf. Fig. 3): (1) the reaction occurs at an interface between two immiscible liquid phases (typically an oil/water interface); (2) at least one of the products from the interfacial reaction has the capability to accelerate the reaction rate, i.e., reaction is autocatalytic; and (3) reaction rate is affected by the change(s) of physical properties (typically, emergence of supramolecular aggregates) rather than by the changes in the activation energy of the reaction. In the following, we discuss a general biphasic autocatalytic reaction and the accompanied phenomena such as self-generation, introduce techniques to characterize this dynamic process, and report on some potential reactive systems.
There are three main events during the course of a biphasic physical autocatalytic reaction near an oil-water interface: (i) production of molecules containing both hydrophobic and hydrophilic components (i.e., hydrotropes, surfactants, lipids, etc.), (ii) emergence of aggregates, and (iii) acceleration of the forward reaction rate. These molecules preferentially distribute at the oil-water interface, reducing the surface tension. When their concentration reaches a critical aggregation concentration (CAC), they undergo self-assembly to form supramolecular aggregates. This stage, the time between the distributing at the interface and reaching the CAC, is denoted as the lag phase. As the aggregates form, insoluble reactive species are bonded and/or trapped within the aggregates and is followed by "phase transfer." The increase in both the local concentration of reactants and the reactive interface area accounts for the greatly enhanced reaction rate. In short, the formation of aggregates causes a considerable enhancement in solubilization of otherwise insoluble reactive species and leads to an increased reaction rate, i.e., autocatalysis. Direct consequences of an enhanced reaction rate near an oil-water interface are (1) an increase in the size of molecules and aggregates over time, (2) morphology transition, and (3) new aggregates emerge via division process (also referred to as self-reproduction, selfreplication, self-propagation, and autopoiesis)..$^{[62-64]}$

Although biphasic physical autocatalytic reactions near immiscible oil-water interfaces have been studied extensively for small-molecular systems like surfactants and lipids, similar studies for polymerizations have not been attempted 
so far. However, a number of polymerization-induced selfassembly (PISA) studies have reported self-generating polymeric vesicles ${ }^{[6]}$ in miscible solvent mixtures. These include nano-objects containing monomethoxy poly(ethylene glycol)poly(glycidyl methacrylate) (mPEG-PGMA) diblock copolymer synthesized by photo-initiated RAFT-mediated PISA conducted in ethanol/water mixture. In this case, a fivefold increase in the rate of polymerization was observed following the lag phase. ${ }^{[20]}$ In the case of miscible solvents, competing interactions among polymers and solvent pairs play an important role in affecting the CAC and the lag phase via the concept of co-non-solvency. ${ }^{[65]}$ Autocatalysis was speculated to be a result of the solvation of the GMA monomers by the formed PGMA chains, leading to high monomer concentration in the cores of micelles. Similar results were obtained for RAFTmediated PISA with monomer, 2-hydroxypropyl methacrylate (HPMA), and the poly(ethylene glycol) (PEG) macromolecular chain transfer agent (macro-CTA). ${ }^{[66]}$ In these studies, ${ }^{[20]}$ water content in ethanol-water mixtures was found to affect polymerization kinetics significantly. The underlying cause of this effect was found to be an altered GMA monomer partition within micelles. In another study, the concentration of macromolecular RAFT agent was found to affect the lag phase in the autocatalytic behavior. ${ }^{[67]}$ Taking poly(2-ethylhexyl acrylate)trithiocarbonate (P2EHA-TTC) as a macro-CTA, an amphiphilic block copolymer with poly(methyl acrylate) (PMA) was synthesized via free-radical dispersion polymerization in isododecane, which could form PMA particles. Furthermore, P2EHA segments acted as a steric stabilizer in the final particle. Therefore, the concentration of P2EHA could alter the time length of the lag phase in the plot of monomer conversion against time. Specifically, higher macromolecular RAFT agent's concentration corresponded to a more extended lag phase. Along with concentration, reaction temperature has also been used to influence the polymerization progress. ${ }^{[68]} \mathrm{A}$ large focus in the field of PISA ${ }^{[6]}$ has been the use of different reaction systems to synthesize amphiphilic di- or tri-block polymers as a means of obtaining polymer aggregates at high solid content, monitoring the aggregates' morphological changes, and detecting their corresponding properties. Less effort has been devoted to studying kinetics, due in large part to the complexity of the measurements required. Recently, kinetics of RAFT-mediated PISA was studied by producing spherical micelles assembled from poly( $N$-acryloylmorpholine)-b-poly $(N$-acryloylthiomorpholine) (P(NAM-b-NAT)). ${ }^{[69]}$ Remarkably different from other reported PISA, which showed accelerated polymerization in the mid-course when micellar aggregates emerged, unnoticeable acceleration of polymerization was reported.

Characterization of reaction kinetics near an oil-water interface has been made possible due to advances in optical microscopy and other interface-sensitive techniques. For example, autocatalytic growth-to-deformation/division of supramolecular aggregates has been extensively investigated ${ }^{[6,60,70]}$ using microscopy. In particular, Veronese and Luisi ${ }^{[71]}$ counted the giant vesicles (1-6 microns in size verified using a freeze-fracture electron microscope) with an optical microscope. Differential interference contrast microscopy and fluorescence microscopy (especially confocal microscopy) are also employed for the same purpose ${ }^{[62,70]}$ However, reactions such as hydrolysis of esters and thiol-ene reaction normally lead to the formation of nano-sized micellar self-assemblies, which are challenging to be directly visualized through conventional optical microscopes. For nanoscale systems, cryogenic electron microscopy has been used. Recently, an alternative optical microscopic technique - interferometric scattering microscopy (iSCAT) was extensively reported by Fletcher and collaborators for studying biphasic physical autocatalysis. ${ }^{[63,72]}$ iSCAT allows direct visualization of nano-sized micelles as well as vesicles. As compared to the thiol in the first reported autocatalytic thiol-ene reaction ${ }^{[73]}$ (Fig. 4), a thiol with the longer chain was selected for forming larger micelles (RH $\sim 3 \mathrm{~nm}$ ), which could reliably be detected by iSCAT. ${ }^{[63]}$ Biphasic thiol-X reactions either on the microscope coverslip or under traditional batch conditions were observed in situ by this label-free microscopic technique,

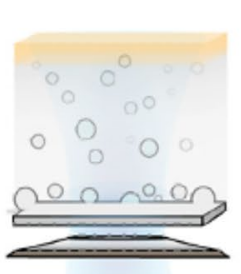

a

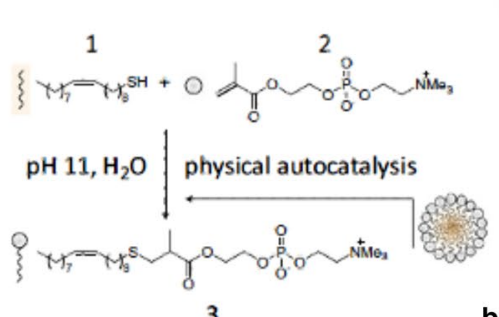

3
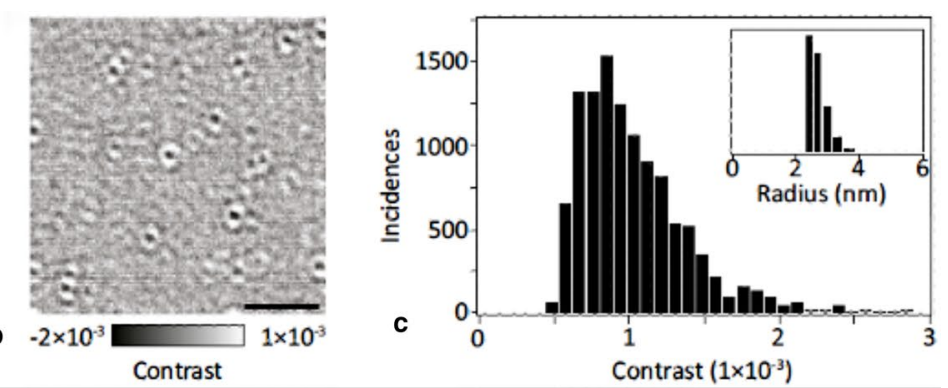

Figure 4. Visualizing physical autocatalysis by an optical microscopy technique, called interferometric scattering microscopy (iSCAT). (a) Schematic of a biphasic reaction between a water-soluble molecule (2) with neat water-insoluble molecule (1) carried out on a microscope coverslip. iSCAT relies on illuminating the sample with a coherent light source and imaging the reflected and backscattered light from the sample. (b) Representative differential iSCAT image of single micelles of molecule (3) bound to microscope cover glass after subtraction of the static scattering background. (Scale bar: 2 microns.) (c) iSCAT contrast histogram of a sample of (3) in water. Inset shows dynamic light scattering number distribution of (3) (1 mM). Adapted from Ref. 63 with permissions. 
which relies on the induced scattering properties changing as the result of the non-specific binding of aggregates to the glass surface. iSCAT observations were corroborated with measurements acquired from fluorescence spectrometry ${ }^{[72]}$ as well as ultra-performance liquid chromatography (UPLC) ${ }^{[74]}$ More importantly, iSCAT exhibited decisive advantages (due to its high sensitivity) over dynamic light scattering (DLS) for allowing verification of the nucleation stage as the reaction proceeded. During iSCAT, multiple binding events may cause undercounting when the concentration of aggregate particles is high, and iSCAT is not able to visualize the growth of formed vesicles. Nevertheless, given the amenability to elucidate the mechanism of self-assembly reaction, focused attention to the development of iSCAT as a means of understanding of PISA is warranted. In addition to characterization of morphologies, measurements probing kinetics of autocatalysis have been also executed. To this end, sophisticated methods like an ensemble of spectrophotometric techniques (UV-Vis, FTIR), NMR spectroscopy, and others have been extensively used. For example, in Ref. 73, the authors confirmed the autocatalytic thiol-ene reaction occurred via micellar autocatalysis by using NMRbased diffusion ordered spectroscopy (DOSY). With recent advances in instrumentation, we envision in situ interfacesensitive characterization tools such as advanced vibrational spectroscopies (i.e., sum-frequency generation spectroscopy (SFG), infrared reflection absorption spectroscopy (IRRAS), two-dimensional infrared spectroscopy) to play important roles in monitoring biphasic systems.

\section{Prospective direction: self-generating polymeric micelles, vesicles from reactions near oil-water interfaces}

In contrast to self-generating micelles and vesicles based on surfactants and lipids, autopoietic polymer-based systems are not that well researched despite several advantages of using polymers. These advantages include robust polymer-membrane properties and an abundant material selection. One primary significance for the autopoietic polymer-based system is to give insights into the origin of life. In addition, polymerization and depolymerization proceeding via biphasic physical autocatalysis will bring new vigor to explore fascinating interfacial phenomena and develop novel applications. A wide range of well-established techniques, especially time-resolved in situ techniques, opens the door to more in-depth characterization. Based on studies probing autocatalysis in lipids and surfactants, a variety of factors can impact similar reactions in the presence of polymers. For example, salting-in and salting-out effect in charged reactive species can affect kinetics of these reactions and remain largely unexplored in the context of polymers. Self-generating polymeric micelles and vesicles have various applications in addition to mimicking functional artificial cells. These applications include drug delivery in an autonomous fashion, model systems for studying membrane rearrangement, morphology dynamics, and other subjects in membrane physics. For these applications, various factors ${ }^{[61]}$ such as reaction parameters, medium properties, and chemical properties of reactive components are expected to influence the selfgeneration progress. As the integration of information carriers (i.e., proteins, DNA) and functionalities (i.e., enzymatic reaction) into self-generating vesicles for establishing functional artificial cells is still a very active area of research, similar works using polymers can provide some insights into designing artificial protocells. In addition, we expect frequent usage of in situ and surface-sensitive characterization techniques such as SFG for probing biphasic physical autocatalytic systems. Furthermore, direct visualization of the nucleation-to-structure transition, and identifying reaction pathways in the interfacial regime are interesting directions for future studies.

\section{Prospective direction: biphasic autocatalysis for polymeric membranes}

Biphasic autocatalysis is quite similar to an interfacial polymerization based on a polycondensation reaction between nucleophiles and electrophiles, in which electrophiles are dissolved in an organic phase. The polycondensation reaction is confined to the organic/aqueous interface due to the reactants' limited diffusion in one of the phases. However, as the reaction progresses, low permeability of synthesized polymers near the interface impedes the reaction due to its effects on the concentration of reactants near the organic/aqueous interface. In other words, in contrast to biphasic physical autocatalysis, neither reactive species solubility in reverse phase nor reactive surface area are able to accelerate the forward reaction rate as the interfacial reaction proceeds. These effects are reflected by a sharp deceleration in the growth of the polymer film (or membrane) thickness over time. Here, we will not review interfacial polymerization, but we refer interested readers to several comprehensive reviews ${ }^{[75,76]}$ on this topic. Here, we discuss the relevance of biphasic autocatalysis for polymeric membranes.

It is noteworthy to recall that the main types of polymers synthesized from interfacial polymerization are polyamides, polyurethanes, polyesters, and polyimides, to list a few. ${ }^{[75]}$ In order to autoaccelerate synthesis of membranes based on copolymers, parameters appearing in a reaction-diffusion process (such as in RAFT-mediated PISA) needs to be considered. These parameters include solubility, diffusivity, and reactivity of reactive species. An example is shown in Fig. 5, which is based on an interfacial polymerization between piperazine (activator) and trimesoyl chloride (inhibitor) occurring on top of polysulfone support, where inhibitor could poorly solubilize in the aqueous phase. ${ }^{[77]}$ This led to the diffusion-controlled polyamide membrane formation proceeding on the organic side. Intriguingly, membranes with Turing structures were produced using porous supports when poly(vinyl alcohol) (PVA) was added into the aqueous phase. This was interpreted by a created appropriate difference between the diffusion coefficient of the separated activator and inhibitor in a system far from thermodynamic equilibrium. If an interfacial polymerization 


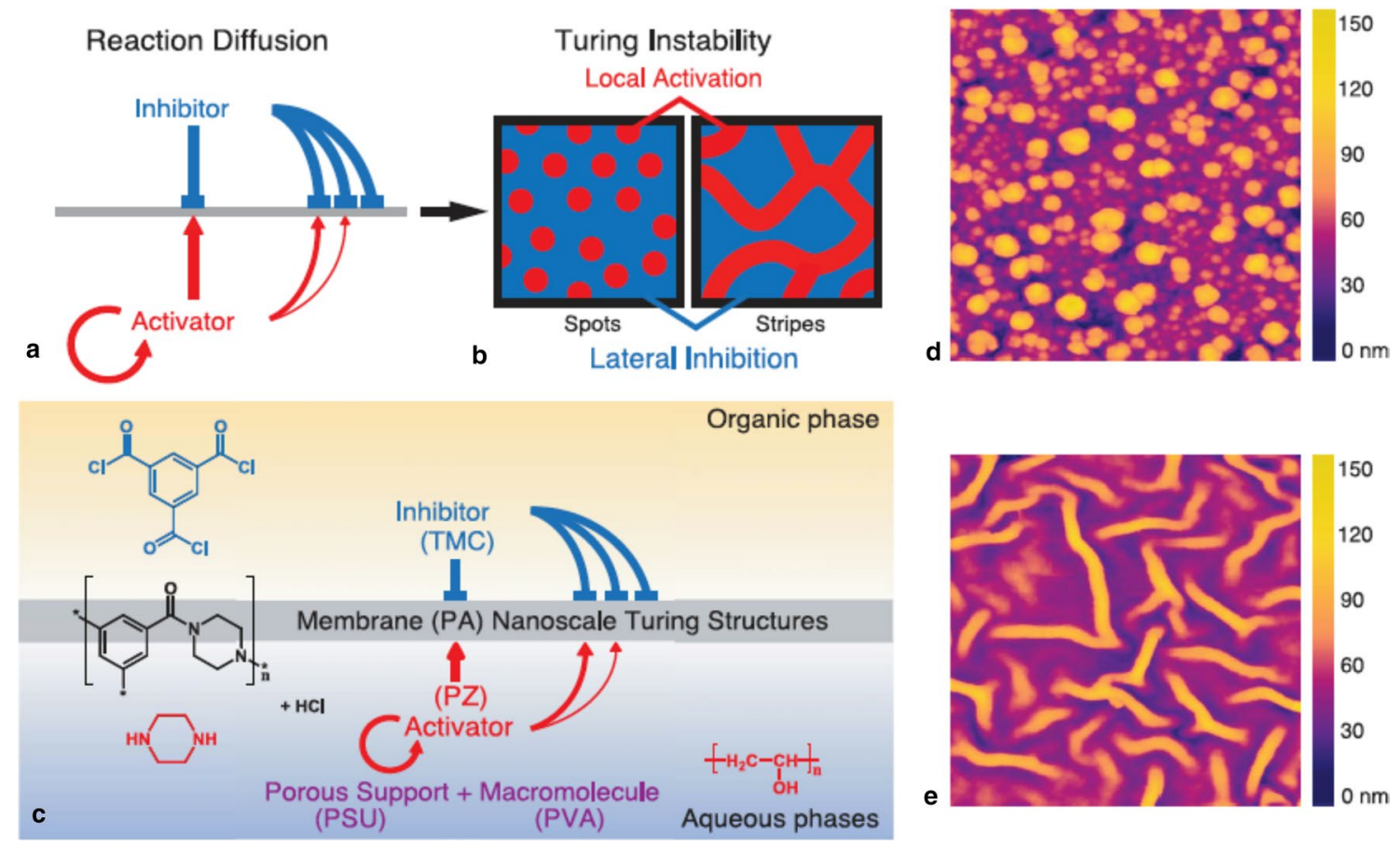

Figure 5. Turing-type structures in interfacial polymerization. (a) Schematic diagram of activator-inhibitor interaction in a reaction-diffusion process. Reactions leading to Turing structures rely on competing activation (red) and inhibition (black) kinetic pathways. (b) Spatial representation of local activation and lateral inhibition. In two dimensions, Turing structures generally consist of spots or stripes. (c) Schematic illustration of interfacial polymerization Turing system. The inhibitor, trimesoyl chloride (TMC), is dissolved in the organic phase (top), and the activator, piperazine (PZ), and the macromolecule poly(vinyl alcohol) (PVA) are dissolved in the aqueous phases (bottom). The polyamide (PA) membrane with nanoscale Turing structures forms on the porous support (PSU). (d and r) AFM topography images of the Turing-type PA membranes. Bright yellow and orange regions correspond to the formed solid-state nanoscale Turing structures. Initial concentrations for nanoscale spots (d) are $[\mathrm{TMC}]=6 \mathrm{mM},[\mathrm{PZ}]=28 \mathrm{mM}$, and $[\mathrm{PVA}]=12 \mathrm{mM}$, and for nanoscale stripes (e), $[\mathrm{TMC}]=8$ $\mathrm{mM},[\mathrm{PZ}]=23 \mathrm{mM}$, and $[\mathrm{PVA}]=32 \mathrm{mM}$. Scan area is 2 microns by 2 microns. Adapted from Ref. 77 with permissions.

were to be executed in the presence of surfactants, ${ }^{[78]}$ which can undergo biphasic autocatalysis (i.e., hydrolysis of surfactant precursors, imine condensation, thiol-X reactions) then the structure of polymeric membranes can be altered significantly by controlling autocatalytic behavior. Therefore, coupling autocatalytic reactions and interfacial polymerization can be used as an alternative route to alter materials' structure and functional properties. As a result, we envisage highly porous membranes, three-dimensional objects, and other interesting structures produced using biphasic autocatalysis.

\section{Modeling of autocatalysis}

Transition state theory (TST) developed by Eyring, ${ }^{[79]}$ Evans, Polanyi ${ }^{[80]}$, and Wigner ${ }^{[81]}$ has become a cornerstone in determining reaction rates from first principles. However, local equilibrium condition with activated complex in TST only allows justification to near-equilibrium processes in a description of reaction rates. Furthermore, application of TST to polymers has been hindered by the enormous computational cost of modeling long chains and the large number of degrees of freedom required to sample various activated complexes. Alternative approaches based on reactive force fields ${ }^{[82]}$ have been developed to simulate polymeric systems at larger length and longer time scales. For the most of the autocatalytic reactions discussed in the previous two sections, which are either diffusion or electrostatic limited, these simulation approaches can only provide activation barriers for known reaction pathways. In order to simulate the effects of diffusion and electrostatics in solutions and melts, continuum reaction-diffusion models based on kinetic equations have been developed. For simulating chemical reactions in polymers, multi-scale models based on classical molecular dynamics have also been developed. These models have the potential to be made more accurate at the expense of computational cost by incorporating information about the reactions using quantum mechanical methods. Recently, models for reaction kinetics in a batch and flow reactor have been developed by using neural networks. In this section, we discuss these methods in terms of their applications in modeling reactions in polymeric systems and their extensions for modeling different aspects of autocatalysis. 


\section{Prospective direction: extensions of reaction- diffusion models}

Turing ${ }^{[7]}$ showed that near an instability for a two component system undergoing reaction and diffusion, six different cases may arise and in the most important case, stationary waves arise. The latter is known as Turing patterns and has been discussed in the previous section in relation to polyamide membranes (cf. Fig. 5) synthesized using interfacial polymerization. In contrast, this instability is completely ignored in the models describing autoacceleration in free-radical polymerization. Such models (discussed in "Autocatalysis in kinetics of polymerization: breakdown of equal reactivity hypothesis" section) focus on connecting rate constants to diffusion constants of reactive free radicals, polymer chain lengths, entanglements, and concentration of the chains. A major focus of the theoretical works related to reactions in polymers has been on the diffusion-controlled reactions. ${ }^{[83,84]}$ Extensions of these works to study effects of fluid flow and electrostatics are required to gain insights into autocatalysis. Efforts to include effects of fluid flow ${ }^{[19]}$ and electrostatics ${ }^{[39,85,86]}$ on reactions have been reported. However, these models have been developed for dilute reacting formulations and they need to be extended to concentrated solutions in order to be applicable for modeling autocatalysis. An attractive approach to extend such models for understanding autocatalysis is to consider reactions using Lotka-Volterra equations ${ }^{[12,13]}$ (also known as predator-prey equations). By modeling reactions using Lotka-Volterra equations and considering diffusion of various chemical species, heterogeneities and chemical instabilities can be captured by these models. However, we are not aware of any such extended model applied to study either polymerization or depolymerization. Hence, for developing a detailed understanding of autocatalysis in polymeric systems and pattern formation, reaction-diffusion models based on Lotka-Volterra equations need to be developed.

\section{Prospective direction: reactive simulations based on molecular dynamics (classical and quantum) and Monte-Carlo methods}

Multi-scale molecular dynamics simulation protocols involving chemical reactions have been used to model living radical polymerization $^{[87]}$ and dynamic bond swaps in covalent adaptive polymer networks such as vitrimers. ${ }^{[88,89]}$ These simulations are multi-scale in a sense that the reacting species are either described in full atomistic details or in a coarse-grained manner, where a group of atoms are grouped together (e.g., at the monomer or the Kuhn segment level). Furthermore, these molecular dynamics simulations involve classical force fields that describes pairwise, bond, angle, and dihedral interactions. Integrated into these methods are Monte-Carlo moves to model chemical reactions, where rates of reactions are estimated from an Arrhenius-type equation. The activation energies and frequency of Monte-Carlo moves determine the reaction rates. In principle, the same simulation methods and protocols can be used to model autocatalytic depolymerization. This type of method, where electron densities are neglected, allows for longer length and timescale simulations that can probe the effects of viscoelasticity inherent to polymers. Such hybrid simulations involving classical molecular dynamics and Monte-Carlo moves enable direct comparisons with experiments using scattering, rheology, or size exclusion chromatography measurements. Furthermore, the hybrid simulations can be complemented by more accurate quantum chemistry methods, e.g., those based on density functional theory (DFT), by providing the model with accurate estimates of activation energies and reaction rates.

Classical molecular dynamics coupled with Monte-Carlo are ideally suited for simulations of several thousands of atoms. The quality of molecular dynamics simulations critically depends on the accuracy of force fields employed. Choice of functional forms and parameters of force fields is crucial to ensure faithful modeling of physico-chemical processes and reproducing experimental measurements. Popular force fields such as CHARMM, ${ }^{[90]} \mathrm{AMBER}^{[91]}$, and GROMOS ${ }^{[92]}$ used in simulations of liquids and biological systems suffer from poor description of bond breaking and formation in which the atomic connectivity changes due to chemical processes. This has been addressed by introducing reactive bond order potentials in methods such as reaxFF ${ }^{[82]}$ and AIREBO-M. ${ }^{[93]}$ The bond order is an empirical function and its values describe the topology of chemical bonding network (single, double, or triple bonding) and coordination of atoms based on the local environment of a given atom. It is calculated from atomic coordinates and used along coordinates to compute energy and forces for molecular dynamics. Reactive force fields have been successful in modeling chemical processes that involve changes in the covalent bonding of hydrocarbons and the condensed phases ${ }^{[93]}$; are particularly promising for modeling chemistries relevant to polymer upcycling.

In general, force fields used in classical molecular dynamics simulations represent an estimation of the ground-state electronic structure energy within the Born-Oppenheimer approximation for potential energy surfaces. The optimization of parameters used in force fields can be done using high-level $\mathrm{ab}$ initio calculations and experimental data to ensure that the predicted observables are accurate and reproduce molecular properties such as crystallographic structures, vibrational frequencies, and thermodynamics. The primary shortcomings of molecular dynamics based on parametrized force fields are the limited applicability to modeling processes involving electronically excited states, such as photo-reactions, redox and field-induced reactions, proton-coupled electron reactions, and charge transfer processes. Such processes generally require explicit treatment of electronic structure and may often need time-dependent quantum mechanical treatment. A natural framework for such simulations is through ab initio molecular dynamics methods.

In the ab initio molecular dynamics-based approaches, the issue of parametrization is not present since the forces that govern the dynamics of the nuclei are calculated directly from the 
electronic structure theory evaluated on-the-fly at each time step. The choice of the specific electronic structure theory is non-trivial and also requires expertise. These ab initio approaches also allow one to go beyond ground-state electronic structure and simulations of chemistries involving electronically excited states and non-adiabatic processes when employing time-dependent quantum dynamical methods. Example applications include non-equilibrium charge transfer, ${ }^{[94]}$ as well as electron beam and electric field-induced chemical transformation ${ }^{[95]}$ of materials.

The main limitation of the ab initio methods is the computational cost of electronic structure, usually a DFT method. The computational time required for a single DFT calculations scales cubically with the system size due to the time cost of the matrix diagonalization. That is, doubling the number of atoms will typically lead to eight-fold increase in the computer time required for simulation of a given number of time steps. Whereas the conventional DFT method is a workhorse for a static electronic structure calculations of hundreds to thousands of atoms, but their high computational cost makes DFT impractical for routine $\mathrm{ab}$ initio molecular dynamics of systems consisting more than a few hundred atoms. Semi-empirical and approximate DFT methods based on tight-binding parametrization, such as density functional tight binding ${ }^{[96]}$ (DFTB), become critical for ab initio molecular dynamics of large systems. The DFTB is an approximate DFT method in which only valence electrons are treated quantum mechanically while all core electrons and nuclei are approximated via pairwise interatomic repulsive potential. For a typical DFTB-based molecular dynamics with 1000 atoms, over $90 \%$ of computer time is spend on diagonalization and on other linear algebra matrix-matrix operation. These simplifications allow to extend routine application of $\mathrm{ab}$ initio methods to molecular systems consisting a few thousands of atoms. A more detailed overview of DFTB method can be found elsewhere. ${ }^{\text {[96] }}$

Although these quantum mechanical methods are accurate and do not require any parameterization, applications of these methods to simulate polymers with $\gg 10,000$ atoms require huge computational resources. With the currently available computational resources, these methods can be used to compute activation barriers for reactions and study probabilities of different reaction pathways by computing underlying potential energy surfaces. With rise in computational resources, it is expected that such quantum mechanical methods based on ab initio molecular dynamics will play an important role in studying mechanisms of reactions by simulating different reaction pathways.

\section{Prospective direction: machine learning for automated reactions and integration with multi-scale models}

Within the last decade, reaction and chemical characterization processes in batch and flow reactors have been optimized using neural networks and automated workflows. Both, single ${ }^{[97]}$ and multi-step ${ }^{[98]}$ reactions, such as those involving nitration, hydrolysis, and hydrogenation have been optimized in flow reactors coupled with in situ chemical characterization via
NMR, UV-VIS, and FTIR. Similar works on reactions following first-order kinetics have appeared and extension of such efforts to autocatalysis exhibiting deviations from first-order kinetics are expected in the near future. Similar advances in automating polymerization reactions ${ }^{[99]}$ using microfluidics have been made and we expect the use of neural networksbased surrogate model developments in future. These advances in coupling automated synthesis to machine learning algorithms will require more accurate models for reaction kinetics. Additionally, bringing new insights into the effects of fluid flow, temperature, and catalyst in affecting autocatalysis will be essential. Integration of machine learning algorithms with multi-scale models for homogeneous reactions ${ }^{[100]}$ has been attempted. For using such algorithms to be useful for studying autocatalysis, they must be extended to include heterogeneity, bifurcation (signature of instability), and non-uniqueness of correspondence between reaction mechanisms and mathematical models. Specifically, neural networks trained on new reaction-diffusion models based on Lotka-Volterra equations, as discussed in "Prospective direction: extensions of reaction-diffusion models" section, can be used to study autocatalysis in automated reactions. These neural networks can be used to not only learn about the non-linear kinetics of reactions but also study the onset of autoacceleration and its origin. This is an interesting direction for future research.

\section{Summary of proposed research directions}

Heterogenity, non-linear kinetics, and the inherent far-fromequilibrium nature of autocatalysis require a concerted effort involving in situ studies of reactions, multi-scale models, and machine learning algorithms in order to garner insights into the mechanisms of autoacceleration, underlying instabilities, and their effects on various patterns and physical properties. In this article, we have proposed a number of prospective directions to pursue, which are technologically relevant and fundamentally important. These directions involve the following:

(1) Development of efficient chemical upcycling protocols by autoaccelerating depolymerization reactions. For developing such protocols, understanding autocatalysis in the kinetics of free-radical depolymerization, especially in the absence of solvent, is a key direction of future research. In particular, studies focused on understanding mechanism and kinetics of photo-oxidation in amorphous and semicrystalline polymers will play a pivotal role in designing the next-generation polymers for a sustainable circular economy.

(2) Autocatalysis has been studied extensively in the presence of solvent, where reactions are controlled by diffusion. In the presence of explicit charges, effects of electrostatics on rate of autoacceleration need to be studied in detail. Such studies are vital for developing faster polymeriza- 
tion schemes of charged polymers as well as acid-base catalyzed depolymerization reactions.

(3) Development of in situ characterization tools for understanding effects of radiations on depolymerization and polymer degradation in semi-crystalline polymers. These tools are based on electron microscopy coupled with pulsed laser systems, which can provide insights into kinetics of reactions with a temporal resolution of femtosecond. In particular, studies showing connections between the loss of crystallinity and depolymerization events are recommended in order to understand mechanisms of polymer degradation in solvent-free polymeric materials.

(4) With advances in using fluid flow for various polymerization schemes, controlling depolymerization reaction rates by fluid flow is an interesting direction of research. In particular, automation of polymerization and depolymerization reactions using microfluidics coupled with in situ chemical characterization via NMR, UV-VIS, and FTIR is a promising direction, which can lead to commercialization of certain reactions. Furthermore, integration of automated synthesis with more accurate multi-scale and neural network-based surrogate models can provide unprecedented insights into mechanisms of reactions.

(5) Autocatalysis near oil-water interfaces can be realized due to changes in physical properties of products during the course of a reaction. These reactions depend on critical aggregation number of products and can be harnessed to create set-ups producing self-generating polymeric micelles and vesicles from reactions near oil-water interfaces. However, mechanism of autocatalysis and trapping of various functional cargo in these micelles and vesicles need to be studied in detail in order to create artificial cells.

(6) Pattern formation due to chemical instabilities in biphasic autocatalysis can be harnessed to generate polymeric membranes with different morphologies. Such pattern formation due to reactions near oil-water interfaces needs to be studied in detail for charged homopolymers and copolymers in order to generate the next generation of gas as well as water desalination polymeric membranes.

(7) Multi-scale models bridging reaction-drift-diffusion models with molecular reactive simulations based on molecular dynamics (classical and quantum) and MonteCarlo methods need to be developed in order to understand roles of different reaction pathways on the onset of autocatalysis and resulting pattern formation. Lastly, machine learning-based surrogate model development has the potential to accelerate the multi-scale model building and comparisons with the experiments.

These directions should be pursued in order to develop innovative routes to the next generation of smart and active materials, vital to a sustainable circular economy.

\section{Acknowledgments}

This research was conducted at the Center for Nanophase Materials Sciences, which is a DOE Office of Science User Facility.

\section{Declarations}

\section{Conflict of interest}

The authors declare that they have no conflict of interest.

\section{Open Access}

This article is licensed under a Creative Commons Attribution 4.0 International License, which permits use, sharing, adaptation, distribution and reproduction in any medium or format, as long as you give appropriate credit to the original author(s) and the source, provide a link to the Creative Commons licence, and indicate if changes were made. The images or other third party material in this article are included in the article's Creative Commons licence, unless indicated otherwise in a credit line to the material. If material is not included in the article's Creative Commons licence and your intended use is not permitted by statutory regulation or exceeds the permitted use, you will need to obtain permission directly from the copyright holder. To view a copy of this licence, visit http://creativecommons. org/licenses/by/4.0/.

\section{References}

1. S.A. Kauffman, Autocatalytic sets of proteins. J. Theor. Biol. 119, 1 (1986)

2. W. Hordijk, A history of autocatalytic sets. Biol. Theory 14, 224 (2019)

3. J.A. Pojman, V.M. llyashenko, A.M. Khan, Free-radical frontal polymerization: self-propagating thermal reaction waves. J. Chem. Soc. Faraday Trans. 92, 2825 (1996)

4. S. Biria, P.P. Malley, T.F. Kahan, I.D. Hosein, Optical autocatalysis establishes novel spatial dynamics in phase separation of polymer blends during photocuring. ACS Macro Lett. 5, 1237 (2016)

5. A.I. Hanopolskyi, V.A. Smaliak, A.I. Novichkov, S.N. Semenov, Autocatalysis: kinetics. mechanisms and design. ChemSystemsChem 3, e2000026 (2021)

6. S. Pearce, J. Perez-Mercader, PISA: construction of self-organized and selfassembled functional vesicular structures. Polym. Chem. 12, 29 (2021)

7. S. Kondo, T. Miura, Reaction-diffusion model as a framework for understanding biological pattern formation. Science 329, 1616 (2010)

8. Y. LeCun, Y. Bengio, G. Hinton, Deep learning. Nature 521, 436 (2015)

9. F. Simini, Nanoscale artificial intelligence: creating artificial neural networks using autocatalytic reactions. arXiv preprint arXiv:1602.09070 (2016)

10. R. Batra, L. Song, R. Ramprasad, Emerging materials intelligence ecosystems propelled by machine learning. Nat. Rev. Mater. 1-24 (2020)

11. D.J. Audus, J.J. de Pablo, Polymer informatics: opportunities and challenges. ACS Macro Lett. 6, 1078 (2017)

12. F.C. Frank, On spontaneous asymmetric synthesis. Biochim. Biophys. Acta 11, 459 (1953)

13. P.F. Stadler, W. Fontana, J.H. Miller, Random catalytic reaction networks Physica D 63, 378 (1993)

14. R. Norrish, R. Smith, Catalysed polymerization of methyl methacrylate in the liquid phase. Nature 150, 336 (1942)

15. V.E. Trommsdorff, H. Köhle, P. Lagally, Zur polymerisation des methacrylsuremethylesters1. Die Makromolekulare Chem. 1, 169 (1948)

16. G.A. O'Neil, M.B. Wisnudel, J.M. Torkelson, A critical experimental examination of the gel effect in free radical polymerization: Do entanglements cause autoacceleration?. Macromolecules 29, 7477 (1996) 
17. G. Grause, M.F. Chien, C. Inoue, Changes during the weathering of polyolefins. Polym. Degrad. Stab. 181, 109364 (2020)

18. M. Shen, M.L. Robertson, Degradation behavior of biobased epoxy resins in mild acidic media. ACS Sustain. Chem. Eng. 9, 438 (2021)

19. P. Schuster, What is special about autocatalysis?. Monatshefte für ChemieChem. Mon. 150, 763 (2019)

20. J. Tan, D. Liu, C. Huang, X. Li, J. He, Q. Xu, L. Zhang, Photoinitiated polymerization-induced self-assembly of glycidyl methacrylate for the synthesis of epoxy-functionalized block copolymer nano-objects. Macromol. Rapid Commun. 38, 1700195 (2017)

21. T.J. Tulig, M. Tirrell, Molecular theory of the Trommsdorff effect. Macromolecules 14, 1501 (1981)

22. P.J. Flory, Molecular size distribution in linear condensation polymers1. J. Am. Chem. Soc. 58, 1877 (1936)

23. D.S. Achilias, Macromol. Theory Simul. 16, 319 (2007)

24. B. Oshaughnessy, J. Yu, Autoacceleration in free radical polymerization. Phys. Rev. Lett. 73, 1723 (1994)

25. J.M. Nölle, S. Primpke, K. Müllen, P. Vana, D. Wöll, Diffusion of single molecular and macromolecular probes during the free radical bulk polymerization of MMA-towards a better understanding of the Trommsdorff effect on a molecular level. Polym. Chem. 7, 4100 (2016)

26. P. Zhan, J. Chen, A. Zheng, H. Shi, F. Chen, D. Wei, X. Xu, Y. Guan, The Trommsdorff effect under shear and bulk polymerization of methyl methacrylate via reactive extrusion. Eur. Polym. J. 122, 109272 (2020)

27. P. Zhan, J. Chen, A. Zheng, H. Shi, T. Wu, D. Wei, X. Xu, Y. Guan, Methyl methacrylate-styrene copolymers prepared by utilizing the Trommsdorff effect in an inverted two-stage extruder. Mater. Res. Express 7, 095305 (2020)

28. A.Z. Niu, J. Stellbrink, J. Allgaier, L. Willner, A. Radulescu, D. Richter, B.W. Koenig, R.P. May, L.J. Fetters, An in situ study of the t-butyllithium initiated polymerization of butadiene in d-heptane via small angle neutron scattering and H1-NMR. J. Chem. Phys. 122, 134906 (2005)

29. A. Niu, J. Stellbrink, J. Allgaier, D. Richter, R. Hartmann, G.J. Domski, G.W. Coates, L.J. Fetters, Polymerization of 1-octene by a pyridylamidohafnium catalyst: A SEC, $1 \mathrm{H}$ NMR and small angle neutron scattering study. Macromolecules 42, 1083 (2009)

30. R. Simha, L. Wall, Kinetics of chain depolymerization. J. Phys. Chem. 56, 707 (1952)

31. L. Wall, S. Madorsky, D. Brown, S. Straus, R. Simha, The depolymerization of polymethylene and polyethylene. J. Am. Chem. Soc. 76, 3430 (1954)

32. L.A. Wall, S. Straus, J.H. Flynn, D. McIntyre, R. Simha, The thermal degradation mechanism of polystyrene1. J. Phys. Chem. 70, 53 (1966)

33. M.L. Castejón, P. Tiemblo, J.M. Gómez-Elvira, Photo-oxidation of thick isotactic polypropylene films I. Characterisation of the heterogeneous degradation kinetics. Polym. Degrad. Stab. 70, 357 (2000)

34. P. Kubisa, Application of ionic liquids as solvents for polymerization processes. Prog. Polym. Sci. 29, 3 (2004)

35. I. Vollmer, M.J.F. Jenks, M.C.P. Roelands, R.J. White, T. van Harmelen, P. de Wild, G.P. van der Laan, F. Meirer, J.T.F. Keurentjes, B.M. Weckhuysen, Beyond mechanical recycling: giving new life to plastic waste. Angew. Chem. Int. Ed. 59, 15402 (2020)

36. M. Chen, J.W. Dugger, X. Li, Y. Wang, R. Kumar, K.M. Meek, D.W. Uhrig, J.F. Browning, L.A. Madsen, T.E. Long, B.S. Lokitz, Polymerized ionic liquids: effects of counter-anions on ion conduction and polymerization kinetics. J. Polym. Sci. Part A 56, 1346 (2018)

37. P.J. Scott, G.A. Spiering, Y. Wang, Z.D. Seibers, R.B. Moore, R. Kumar, B.S. Lokitz, T.E. Long, Phosphonium-based polyzwitterions: influence of ionic structure and association on mechanical properties. Macromolecules $\mathbf{5 3}$, 11009 (2020)

38. R.A. Marcus, Reflections on electron transfer theory. J. Chem. Phys. 153, $210401(2020)$

39. L. Onsager, Deviations from Ohm's law in weak electrolytes. J. Chem. Phys. 2, 599 (1934)

40. R. Rinaldi, R. Palkovits, F. Schth, Angew. Depolymerization of cellulose using solid catalysts in ionic liquids. Chem. Int. Ed. 47, 8047 (2008)

41. A. Kamimura, Y. Shiramatsu, T. Kawamoto, Depolymerization of polyamide 6 in hydrophilic ionic liquids, Special Issue: Ionic Liquids in Energy and Environment. Green Energy Environ. 4, 166 (2019)
42. A.V. Dobrynin, M. Jacobs, When do polyelectrolytes entangle? Macromolecules 54, 1859 (2021)

43. A.L. Forster, Z. Tsinas, M. Al-Sheikhly, Effect of irradiation and detection of long-lived polyenyl radicals in highly crystalline ultra-high molar mass polyethylene (UHMMPE) fibers. Polymers 11, 924 (2019)

44. A. Ashfaq, M.C. Clochard, X. Coqueret, C. Dispenza, M.S. Driscoll, P. Ulański, M. Al-Sheikhly, Polymerization reactions and modifications of polymers by ionizing radiation. Polymers 12, 2877 (2020)

45. K. Kobayashi, K. Sakaoku, The changes of polymer crystals due to irradiation with electrons accelerated at various voltages (special issue on electron microscopy). Bull. Inst. Chem. Res. Kyoto Univ. 42, 473 (1965)

46. Z. Zhang, J. Yang, W. Huang, H. Wang, W. Zhou, Y. Li, Y. Li, J. Xu, W. Huang, W. Chiu et al., Cathode-electrolyte interphase in lithium batteries revealed by cryogenic electron microscopy. Matter 4, 302 (2021)

47. M. Sun, C. Azumaya, E. Tse, A. Frost, D. Southworth, K. Verba, Y. Cheng, D. Agard, Practical considerations for using K3 cameras in CDS mode for high-resolution and high-throughput single particle cryo-EM. J. Struct. Biol. 107745 (2021)

48. D.J. Flannigan, A.H. Zewail, 4D electron microscopy: principles and applications. Acc. Chem. Res. 45, 1828 (2012)

49. J.W. Lau, K.B. Schliep, M.B. Katz, V.J. Gokhale, J.J. Gorman, C. Jing, A. Liu, Y. Zhao, E. Montgomery, H. Choe et al., Laser-free GHz stroboscopic transmission electron microscope: components, system integration, and practical considerations for pump-probe measurements. Rev. Sci. Instrum. 91, 021301 (2020)

50. S. Hwang, X. Chen, G. Zhou, D. Su, In situ transmission electron microscopy on energy-related catalysis. Adv. Energy Mater. 10, 1902105 (2020)

51. P. Ye, P.F. Cao, Z. Su, R. Advincula, Highly efficient reversible additionfragmentation chain-transfer polymerization in ethanol/water via flow chemistry. Polym. Int. 66, 1252 (2017)

52. P. Ye, P.F. Cao, Q. Chen, R. Advincula, Continuous flow fabrication of block copolymer-grafted silica micro-particles in environmentally friendly water/ ethanol media. Macromol. Mater. Eng. 304, 1800451 (2019)

53. O.Y. Abdelaziz, C.P. Hulteberg, Lignin depolymerization under continuousflow conditions: highlights of recent developments. ChemSusChem 13, $4382(2020)$

54. R.E. Yardley, A.R. Kenaree, E.R. Gillies, Triggering depolymerization: progress and opportunities for self-immolative polymers. Macromolecules 52, 6342 (2019)

55. R.L. Hartman, Flow chemistry remains an opportunity for chemists and chemical engineers. Curr. Opin. Chem. Eng. 29, 42 (2020)

56. V. Delplace, J. Nicolas, Degradable vinyl polymers for biomedical applications. Nat. Chem. 7, 771 (2015)

57. M.N. Siddiqui, L. Kolokotsiou, E. Vouvoudi, H.H. Redhwi, A.A. Al-Arfaj, D.S. Achilias, Depolymerization of PLA by phase transfer catalysed alkaline hydrolysis in a microwave reactor. J. Polym. Environ. 28, 1664 (2020)

58. J.A. Herman, M.E. Seazzu, L.G. Hughes, D.R. Wheeler, C.M. Washburn, B.H. Jones, Depolymerization of cross-linked polybutadiene networks in situ using latent alkene metathesis. ACS Appl. Polym. Mater. 1, 2177 (2019)

59. S.C. Kosloski-Oh, Z.A. Wood, Y. Manjarrez, J.P. de los Rios, M.E. Fieser, Catalytic methods for chemical recycling or upcycling of commercial polymers. Mater. Horiz. (2021). https://doi.org/10.1039/D0MH01286F

60. P.A. Bachmann, P.L. Luisi, J. Lang, Autocatalytic self-replicating micelles as models for prebiotic structures. Nature 357, 57 (1992)

61. E.A. Post, S.P. Fletcher, Controlling the kinetics of self-reproducing micelles by catalyst compartmentalization in a biphasic system. J. Org. Chem. 84, 2741 (2019)

62. M.D. Hardy, J. Yang, J. Selimkhanov, C.M. Cole, L.S. Tsimring, N.K. Devaraj, Self-reproducing catalyst drives repeated phospholipid synthesis and membrane growth. Proc. Natl. Acad. Sci. 112, 8187 (2015)

63. J. Ortega-Arroyo, A.J. Bissette, P. Kukura, S.P. Fletcher, Visualization of the spontaneous emergence of a complex, dynamic, and autocatalytic system. Proc. Natl. Acad. Sci. 113, 11122 (2016)

64. J.M. Carnall, C.A. Waudby, A.M. Belenguer, M.C. Stuart, J.J.P. Peyralans, S. Otto, Mechanosensitive self-replication driven by self-organization. Science 327, 1502 (2010)

65. D. Mukherji, C.M. Marques, K. Kremer, Polymer collapse in miscible good solvents is a generic phenomenon driven by preferential adsorption. Nat. Commun. 5, 1 (2014) 
66. N.J. Warren, 0.0. Mykhaylyk, D. Mahmood, A.J. Ryan, S.P. Armes, RAFT aqueous dispersion polymerization yields poly (ethylene glycol)-based diblock copolymer nano-objects with predictable single phase morphologies. J. Am. Chem. Soc. 136, 1023 (2014)

67. L. Houillot, C. Bui, C. Farcet, C. Moire, J.A. Raust, H. Pasch, M. Save, B. Charleux, Dispersion polymerization of methyl acrylate in nonpolar solvent stabilized by block copolymers formed in situ via the RAFT process. ACS Appl. Mater. Interfaces 2, 434 (2010)

68. S. Perrier, 50th anniversary perspective: RAFT polymerization a user guide. Macromolecules 50, 7433 (2017)

69. R. Takahashi, S. Miwa, F.H. Sobotta, J.H. Lee, S. Fujii, N. Ohta, J.C. Brendel, K. Sakurai, Unraveling the kinetics of the structural development during polymerization-induced self-assembly: decoupling the polymerization and the micelle structure. Polym. Chem.11, 1514 (2020)

70. R. Wick, P. Walde, P.L. Luisi, Light microscopic investigations of the autocatalytic self-reproduction of giant vesicles. J. Am. Chem. Soc. 117, 1435 (1995)

71. A. Veronese, P.L. Luisi, An autocatalytic reaction leading to spontaneously assembled phosphatidyl nucleoside giant vesicles. J. Am. Chem. Soc. 120, 2662 (1998)

72. M.A. Lebedeva, E. Palmieri, P. Kukura, S.P. Fletcher, Emergence and rearrangement of dynamic supramolecular aggregates visualized by interferometric scattering microscopy. ACS Nano 14, 11160 (2020)

73. A.J. Bissette, B. Odell, S.P. Fletcher, Physical autocatalysis driven by a bond-forming thiol-ene reaction. Nat. Commun. 5, 1 (2014)

74. A.H. Engwerda, J. Southworth, M.A. Lebedeva, R.J. Scanes, P. Kukura, S.P. Fletcher, Coupled metabolic cycles allow out-of-equilibrium autopoietic vesicle replication. Angew. Chem. 132, 20541 (2020)

75. M.J. Raaijmakers, N.E. Benes, Current trends in interfacial polymerization chemistry. Prog. Polym. Sci. 63, 86 (2016)

76. M.Q. Seah, W.J. Lau, P.S. Goh, H.H. Tseng, R.A. Wahab, A.F. Ismail, Progress of interfacial polymerization techniques for polyamide thin film (nano) composite membrane fabrication: a comprehensive review. Polymers 12, 2817 (2020)

77. Z. Tan, S. Chen, X. Peng, L. Zhang, C. Gao, Polyamide membranes with nanoscale Turing structures for water purification. Science 360, 518 (2018)

78. K. Liu, H. Qi, R. Dong, R. Shivhare, M. Addicoat, T. Zhang, H. Sahabudeen, T. Heine, S. Mannsfeld, U. Kaiser et al., n-water surface synthesis of crystalline, few-layer two-dimensional polymers assisted by surfactant monolayers. Nat. Chem. 11, 994 (2019)

79. H. Eyring, The activated complex in chemical reactions. J. Chem. Phys. 3, 107 (1935)

80. M.G. Evans, M. Polanyi, Some applications of the transition state method to the calculation of reaction velocities, especially in solution. Trans. Faraday Soc. 31, 875 (1935)

81. E. Wigner, The transition state method. Trans. Faraday Soc. 34, 29 (1938)

82. A.C.T. van Duin, S. Dasgupta, F. Lorant, W.A. Goddard, ReaxFF: a reactive force field for hydrocarbons. J. Phys. Chem. A 105, 9396 (2001)

83. P. Hänggi, P. Talkner, M. Borkovec, Reaction-rate theory: fifty years after Kramers. Rev. Mod. Phys. 62, 251 (1990)

84. R. Vinu, L.J. Broadbelt, Unraveling reaction pathways and specifying reaction kinetics for complex systems. Ann. Rev. Chem. Biomol. Eng. 3, 29 (2012)
85. R. Kumar, V. Bocharova, E. Strelcov, A. Tselev, I.I. Kravchenko, S. Berdzinski, V. Strehmel, 0.S. Ovchinnikova, J.A. Minutolo, J.R. Sangoro, A.L. Agapov, A.P. Sokolov, S.V. Kalinin, B.G. Sumpter, lon transport and softening in a polymerized ionic liquid. Nanoscale 7, 947 (2015)

86. V. Bocharova, A.L. Agapov, A. Tselev, L. Collins, R. Kumar, S. Berdzinski, V. Strehmel, A. Kisliuk, I.I. Kravchenko, B.G. Sumpter, A.P. Sokolov, S.V. Kalinin, E. Strelcov, Adv. Funct. Mater. 25, 805 (2015)

87. S.K. Ahn, J.M.Y. Carrillo, Y. Han, T.H. Kim, D. Uhrig, D.L. Pickel, K. Hong, S.M. Kilbey, B.G. Sumpter, G.S. Smith, C. Do, Structural evolution of polylactide molecular bottlebrushes: kinetics study by size exclusion chromatography, small angle neutron scattering, and simulations. ACS Macro Lett. 3, 862 (2014)

88. J.B. Wu, S.J. Li, H. Liu, H.J. Qian, Z.Y. Lu, Dynamics and reaction kinetics of coarse-grained bulk vitrimers: a molecular dynamics study. Phys. Chem. Chem. Phys. 21, 13258 (2019)

89. A. Perego, F. Khabaz, Volumetric and rheological properties of vitrimers: a hybrid molecular dynamics and Monte Carlo simulation study. Macromolecules 53, 8406 (2020)

90. S. Patel, C.L. Brooks III., CHARMM fluctuating charge force field for proteins: I parameterization and application to bulk organic liquid simulations. J. Comput. Chem. 25, 1 (2004)

91. L. Yang, C.H. Tan, M.J. Hsieh, J. Wang, Y. Duan, P. Cieplak, J. Caldwell, P.A. Kollman, R. Luo, New-generation amber united-atom force field. J. Phys. Chem. B 110, 13166 (2006)

92. A.P. Eichenberger, W. Huang, S. Riniker, W.F. van Gunsteren, Supra-atomic coarse-grained gromos force field for aliphatic hydrocarbons in the liquid phase. J. Chem. Theory Comput. 11, 2925 (2015)

93. T.C. O'Connor, J. Andzelm, M.O. Robbins, AIREBO-M: A reactive model for hydrocarbons at extreme pressures. J. Chem. Phys. 142, 024903 (2015)

94. J. Jakowski, S. Irle, K. Morokuma, Time-dependent quantum dynamical simulations of $\mathrm{CO} 2$ condensation under extreme conditions. Phys. Chem. Chem. Phys. 14, 6273 (2012)

95. D.B. Lingerfelt, P. Ganesh, J. Jakowski, B.G. Sumpter, Understanding beaminduced electronic excitations in materials. J. Chem. Theory Comput. 16, $1200(2020)$

96. F. Spiegelman, N. Tarrat, J. Cuny, L. Dontot, E. Posenitskiy, C. Martí, A. Simon, M. Rapacioli, Density-functional tight-binding: basic concepts and applications to molecules and clusters. Adv. Phys. X5, 1710252 (2020)

97. C.J. Taylor, M. Booth, J.A. Manson, M.J. Willis, G. Clemens, B.A. Taylor, T.W. Chamberlain, R.A. Bourne, Rapid, automated determination of reaction models and kinetic parameters. Chem. Eng. J. 127017 (2020)

98. P. Sagmeister, R. Lebl, I. Castillo, J. Rehrl, J. Kruisz, M. Sipek, M. Horn, S. Sacher, D. Cantillo, J.D. Williams, C.O. Kappe, Advanced real-time process analytics for multistep synthesis in continuous flow. Angew. Chem. Int. Ed. https://doi.org/10.1002/anie.202016007 (2021)

99. B.A. Rizkin, A.S. Shkolnik, N.J. Ferraro, R.J. Hartman, Combining automated microfluidic experimentation with machine learning for efficient polymerization design. Nat. Mach. Intell. 2, 200 (2020)

100. W. Yang, L. Peng, Y. Zhu, L. Hong, When machine learning meets multiscale modeling in chemical reactions. J. Chem. Phys. 153, 094117 (2020) 